\title{
Unital Compact Homomorphisms between Extended Analytic Lipschitz Algebras
}

\author{
Davood Alimohammadi and Maliheh Mayghani \\ Department of Mathematics, Faculty of Science, Arak University, Arak 38156-8-8349, Iran \\ Correspondence should be addressed to Davood Alimohammadi, d-alimohammadi@araku.ac.ir
}

Received 22 May 2011; Accepted 10 August 2011

Academic Editor: Malisa R. Zizovic

Copyright (c) 2011 D. Alimohammadi and M. Mayghani. This is an open access article distributed under the Creative Commons Attribution License, which permits unrestricted use, distribution, and reproduction in any medium, provided the original work is properly cited.

Let $X$ and $K$ be compact plane sets with $K \subseteq X$. We define $A(X, K)=\left\{f \in C(X):\left.f\right|_{K} \in\right.$ $A(K)\}$, where $A(K)=\{g \in C(X): g$ is analytic on $\operatorname{int}(K)\}$. For $\alpha \in(0,1]$, we define $\operatorname{Lip}(X, K, \alpha)=\left\{f \in C(X): p_{\alpha, K}(f)=\sup \left\{|f(z)-f(w)| /|z-w|^{\alpha}: z, w \in K, z \neq w\right\}<\infty\right\}$ and $\operatorname{Lip}_{A}(X, K, \alpha)=A(X, K) \cap \operatorname{Lip}(X, K, \alpha)$. It is known that $\operatorname{Lip}_{A}(X, K, \alpha)$ is a natural Banach function algebra on $X$ under the norm $\|f\|_{\operatorname{Lip}(X, K, \alpha)}=\|f\|_{X}+p_{\alpha, K}(f)$, where $\|f\|_{X}=\sup \{|f(x)|: x \in X\}$. These algebras are called extended analytic Lipschitz algebras. In this paper we study unital homomorphisms from natural Banach function subalgebras of $\operatorname{Lip}_{A}\left(X_{1}, K_{1}, \alpha_{1}\right)$ to natural Banach function subalgebras of $\operatorname{Lip}_{A}\left(X_{2}, K_{2}, \alpha_{2}\right)$ and investigate necessary and sufficient conditions for which these homomorphisms are compact. We also determine the spectrum of unital compact endomorphisms of $\operatorname{Lip}_{A}(X, K, \alpha)$.

\section{Introduction and Preliminaries}

We let $\mathbb{C}, \mathbb{D}=\{z \in \mathbb{C}:|z|<1\}, \overline{\mathbb{D}}=\{z \in \mathbb{C}:|z| \leq 1\}, \mathbb{D}(\lambda, r)=\{z \in \mathbb{C}:|z-\lambda|<r\}$, and $\overline{\mathbb{D}(\lambda, r)}=\{z \in \mathbb{C}:|z-\lambda| \leq r\}$ denote the field of complex numbers, the open unit disc, the closed unit disc, and the open and closed discs with center at $\lambda$ and radius $r$, respectively. We also denote $\mathbb{D}(0, r)$ by $\mathbb{D}_{r}$.

Let $A$ and $B$ be unital commutative semisimple Banach algebras with maximal ideal spaces $\mathcal{M}(A)$ and $\mathcal{M}(B)$. A homomorphism $T: A \rightarrow B$ is called unital if $T 1_{A}=1_{B}$. If $T$ is a unital homomorphism from $A$ into $B$, then $T$ is continuous and there exists a normcontinuous map $\varphi: \mathcal{M}(B) \rightarrow \mathcal{M}(A)$ such that $\widehat{T f}=\widehat{f} \circ \varphi$ for all $f \in A$, where $\widehat{g}$ is the Gelfand transform $g$. In fact, $\varphi$ is equal the adjoint of $T^{*}: B^{*} \rightarrow A^{*}$ restricted to $\mathcal{M}(B)$. Note that $T^{*}$ is a weak*-weak* continuous map from $B^{*}$ into $A^{*}$. Thus $\varphi$ is a continuous map from $\mathcal{M}(B)$ with the Gelfand topology into $\mathcal{M}(A)$ with the Gelfand topology. 
Let $A$ be a unital commutative semisimple Banach algebra, and let $T$ be an endomorphism of $A$, a homomorphism from $A$ into $A$. We denote the spectrum of $T$ by $\sigma(T)$ and define

$$
\sigma(T)=\{\lambda \in \mathbb{C}: \lambda I-T \text { is not invertible }\} .
$$

For a compact Hausdorff space $X$, we denote by $C(X)$ the Banach algebra of all continuous complex-valued functions on $X$.

Definition 1.1. Let $X$ be a compact Hausdorff space. A Banach function algebra on $X$ is a subalgebra $A$ of $C(X)$ which contains $1_{X}$, the constant function 1 on $X$, separates the points of $X$, and is a unital Banach algebra with an algebra norm $\|\cdot\|$. If the norm of a Banach function algebra on $X$ is $\|\cdot\|_{X}$, the uniform norm on $X$, it is called a uniform algebra on $X$.

Let $A$ and $B$ be Banach function algebras on $X$ and $Y$, respectively. If $\varphi: Y \rightarrow X$ is a continuous mapping such that $f \circ \varphi \in B$ for all $f \in A$ and if $T: A \rightarrow B$ is defined by $T f=f \circ \varphi$, then $T$ is a unital homomorphism, which is called the induced homomorphism from $A$ into $B$ by $\varphi$. In particular, if $Y=X$ and $B=A$, then $T$ is called the induced endomorphism of $A$ by the self-map $\varphi$ of $X$.

Let $A$ be a Banach function algebra on a compact Hausdorff space $X$. For $x \in X$, the map $e_{x}: A \rightarrow \mathbb{C}$, defined by $e_{x}(f)=f(x)$, is an element of $\mathcal{M}(A)$ and is called the evaluation homomorphism on $A$ at $x$. This fact implies that $A$ is semisimple and $\|f\|_{X} \leq\|\widehat{f}\|_{\mathcal{M}(A)}$ for all $f \in A$. Note that the map $x \mapsto e_{x}: X \rightarrow \mathcal{M}(A)$ is a continuous one-to-one mapping. If this map is onto, we say that $A$ is natural.

Proposition 1.2. Let $X$ and $Y$ be compact Hausdorff spaces, and let $A$ and $B$ be natural Banach function algebras on $X$ and $Y$, respectively. Then every unital homomorphism $T: A \rightarrow B$ is induced by a unique continuous map $\varphi: Y \rightarrow X$. In particular, if $X$ is a compact plane set and the coordinate function $Z$ belongs to $A$, then $\varphi=T Z$ and so $\varphi \in B$.

Proof. Let $T: A \rightarrow B$ be a unital homomorphism. Since $A$ and $B$ are unital commutative semisimple Banach algebras, there exists a continuous map $\psi: \mathcal{M}(B) \rightarrow \mathcal{M}(A)$ such that $\widehat{T f}=\widehat{f} \circ \psi$ for all $f \in A$. The naturality of the Banach function algebra $A$ on $X$ implies that the map $J_{A}: X \rightarrow \mathcal{M}(A)$, defined by $J_{A}(x)=e_{x}$, is a homeomorphism and so $J_{A}^{-1}: \mathcal{M}(A) \rightarrow X$ is continuous. Since $B$ is a Banach function algebra on $Y$, the map $J_{B}: Y \rightarrow \mathcal{M}(B)$, defined by $J_{B}(y)=e_{y}$, is continuous. We now define the map $\varphi: Y \rightarrow X$ by $\varphi=J_{A}^{-1} \circ \psi \circ J_{B}$. Clearly, $\varphi$ is continuous. Let $f \in A$. Since

$$
\begin{aligned}
(T f)(y) & =\widehat{T f}\left(e_{y}\right)=(\widehat{f} \circ \psi)\left(J_{B}(y)\right)=\left(\widehat{f} \circ J_{A}\right)(\varphi(y)) \\
& =\widehat{f}\left(e_{\varphi(y)}\right)=e_{\varphi(y)}(f)=f(\varphi(y)) \\
& =(f \circ \varphi)(y),
\end{aligned}
$$

for all $y \in Y$, we have $T f=f \circ \varphi$. Therefore, $T$ is induced by $\varphi$.

Now, let $X$ be a compact plane set, and let $Z \in A$. Then $\varphi=Z \circ \varphi=T Z$, and so $\varphi \in B$. 
Corollary 1.3. Let $X$ be a compact Hausdorff space, and let $A$ be a natural Banach function algebra on $X$. Then every unital endomorphism $T$ of $A$ is induced by a unique continuous self-map $\varphi$ of $X$. In particular, if $X$ is a compact plane set and $A$ contains the coordinate function $Z$, then $\varphi=T Z$ and so $\varphi \in A$.

Definition 1.4. Let $X$ be a compact plane set which is connected by rectifiable arcs, and let $\delta(z, w)$ be the geodesic metric on $X$, the infimum of the length of the arcs joining $z$ and $w$. $X$ is called uniformly regular if there exists a constant $C$ such that, for all $z, w \in X, \delta(z, w) \leq C|z-w|$. sequel.

The following lemma occurs in [1] but it is important and we will be using it in the

Lemma 1.5 (see [1, Lemma 1.5]). Let $H$ and $K$ be two compact plane sets with $H \subseteq \operatorname{int}(K)$. Then there exists a finite union of uniformly regular sets $i n \operatorname{int}(K)$ containing $H$, namely $Y$, and then a positive constant $C$ such that for every analytic complex-valued function $f$ on $\operatorname{int}(K)$ and any $z, w \in H$,

$$
|f(z)-f(w)| \leq C|z-w|\left(\|f\|_{Y}+\left\|f^{\prime}\right\|_{Y}\right) .
$$

Let $X$ be a compact plane set. We denote by $A(X)$ the algebra of all continuous complex-valued functions on $X$ which are analytic on $\operatorname{int}(X)$, the interior of $X$, and call it the analytic uniform algebra on $X$. It is known that $A(X)$ is a natural uniform algebra on $X$.

Let $X$ and $K$ be compact plane sets such that $K \subseteq X$. We define $A(X, K)=\{f \in C(X)$ : $\left.\left.f\right|_{K} \in A(K)\right\}$. Clearly, $A(X, K)=A(X)$ if $K=X$, and $A(X, K)=C(X)$ if $\operatorname{int}(K)$ is empty. We know that $A(X, K)$ is a natural uniform algebra on $X$ (see [2]) and call it the extended analytic uniform algebra on $X$ with respect to $K$.

Let $(X, d)$ be a compact metric space. For $\alpha \in(0,1]$, we denote by $\operatorname{Lip}(X, \alpha)$ the algebra of all complex-valued functions $f$ for which $p_{\alpha, X}(f)=\sup \left\{|f(z)-f(w)| / d^{\alpha}(z, w): z, w \in\right.$ $X, z \neq w\}<\infty$. For $f \in \operatorname{Lip}(X, \alpha)$, we define the $\alpha$-Lipschitz norm $f$ by $\|f\|_{\operatorname{Lip}(X, \alpha)}=\|f\|_{X}+$ $p_{\alpha, X}(f)$. Then $\left(\operatorname{Lip}(X, \alpha),\|\cdot\|_{\operatorname{Lip}(X, \alpha)}\right)$ is a unital commutative Banach algebra. For $\alpha \in(0,1)$, we denote by $\operatorname{lip}(X, \alpha)$ the algebra of all complex-valued functions $f$ on $X$ for which $\mid f(z)$ $f(w) \mid / d^{\alpha}(z, w) \rightarrow 0$ as $d(z, w) \rightarrow 0$. Then $\operatorname{lip}(X, \alpha)$ is a unital closed subalgebra of $\operatorname{Lip}(X, \alpha)$. These algebras are called Lipschitz algebras of order $\alpha$ and were first studied by Sherbert in $[3,4]$. We know that the Lipschitz algebras $\operatorname{Lip}(X, \alpha)$ and $\operatorname{lip}(X, \alpha)$ are natural Banach function algebras on $X$.

Let $(X, d)$ be a compact metric space, and let $K$ be a compact subset of $X$. For $\alpha \in(0,1]$, we denote by $\operatorname{Lip}(X, K, \alpha)$ the algebra of all complex-valued functions $f$ on $X$ for which $p_{\alpha, K}(f)=\sup \left\{|f(z)-f(w)| / d^{\alpha}(z, w): z, w \in K, z \neq w\right\}<\infty$. In fact, $\operatorname{Lip}(X, K, \alpha)=\{f \in$ $\left.C(X):\left.f\right|_{K} \in \operatorname{Lip}(K, \alpha)\right\}$. For $f \in \operatorname{Lip}(X, K, \alpha)$, we define $\|f\|_{\operatorname{Lip}(X, K, \alpha)}=\|f\|_{X}+p_{\alpha, K}(f)$. Then $\operatorname{Lip}(X, K, \alpha)$ under the algebra norm $\|\cdot\|_{\operatorname{Lip}(X, K, \alpha)}$ is a unital commutative Banach algebra. Moreover, $\operatorname{Lip}(X, \alpha)$ is a subalgebra of $\operatorname{Lip}(X, K, \alpha) ; \operatorname{Lip}(X, K, \alpha)=\operatorname{Lip}(X, \alpha)$ if $X \backslash K$ is finite, and $\operatorname{Lip}(X, K, \alpha)=C(X)$ if $K$ is finite. For $\alpha \in(0,1)$, we denote by $\operatorname{lip}(X, K, \alpha)$ the algebra of all complex-valued functions $f$ on $X$ for which $|f(z)-f(w)| / d^{\alpha}(z, w) \rightarrow 0$ as $d(z, w) \rightarrow 0$ with $z, w \in K$. In fact, $\operatorname{lip}(X, K, \alpha)=\left\{f \in C(X):\left.f\right|_{K} \in \operatorname{lip}(K, \alpha)\right\}$. Clearly, $\operatorname{lip}(X, K, \alpha)$ is a closed unital subalgebra of $\operatorname{Lip}(X, K, \alpha)$. Moreover, $\operatorname{lip}(X, \alpha)$ is a subalgebra of $\operatorname{lip}(X, K, \alpha) ; \operatorname{lip}(X, K, \alpha)=\operatorname{lip}(X, \alpha)$ if $X \backslash K$ is finite, and $\operatorname{lip}(X, K, \alpha)=C(X)$ if $K$ is finite. The Banach algebras $\operatorname{Lip}(X, K, \alpha)$ and $\operatorname{lip}(X, K, \alpha)$ are Banach function algebras on $X$ and were first introduced by Honary and Moradi in [5]. 
Let $X$ be a compact plane set. We define $\operatorname{Lip}_{A}(X, \alpha)=\operatorname{Lip}(X, \alpha) \cap A(X)$ for $\alpha \in(0,1]$ and $\operatorname{lip}_{A}(X, \alpha)=\operatorname{Lip}(X, \alpha) \cap A(X)$ for $\alpha \in(0,1)$. These algebras are called analytic Lipschitz algebras. We know that analytic Lipschitz algebras $\operatorname{Lip}_{A}(X, \alpha)$ and $\operatorname{lip}_{A}(X, \alpha)$ under the norm $\|\cdot\|_{\operatorname{Lip}(X, \alpha)}$ are natural Banach function algebras on $X$ (see [6]).

Let $X$ and $K$ be compact plane sets with $K \subseteq X$. We define $\operatorname{Lip}_{A}(X, K, \alpha)=$ $\operatorname{Lip}(X, K, \alpha) \cap A(X, K)$ for $\alpha \in(0,1]$ and $\operatorname{lip}_{A}(X, K, \alpha)=\operatorname{lip}(X, K, \alpha) \cap A(X, K)$ for $\alpha \in(0,1)$. Then $\operatorname{Lip}_{A}(X, K, \alpha)$ and $\operatorname{lip}(X, K, \alpha)$ are closed unital subalgebras of $\operatorname{Lip}(X, K, \alpha)$ and $\operatorname{lip}(X, K, \alpha)$ under the norm $\|\cdot\|_{\operatorname{Lip}(X, K, \alpha)}$, respectively. Moreover, $\operatorname{Lip}_{A}(X, K, \alpha)=$ $\operatorname{Lip}_{A}(X, \alpha)\left[\operatorname{lip}_{A}(X, K, \alpha)=\operatorname{Lip}_{A}(X, \alpha)\right]$ if $K=X$, and $\operatorname{Lip}_{A}(X, K, \alpha)=$ $\operatorname{Lip}(X, K, \alpha)\left[\operatorname{lip}_{A}(X, K, \alpha)=\operatorname{lip}(X, K, \alpha)\right]$ if $\operatorname{int}(K)$ is empty.

The algebras $\operatorname{Lip}_{A}(X, K, \alpha)$ and $\operatorname{lip}(X, K, \alpha)$ are called extended analytic Lipschitz algebras and were first studied by Honary and Moradi in [5]. They showed that the extended analytic Lipschitz algebras $\operatorname{Lip}_{A}(X, K, \alpha)$ and $\operatorname{lip}_{A}(X, K, \alpha)$ under the norm $\|\cdot\|_{\operatorname{Lip}(X, K, \alpha)}$ are natural Banach function algebras on $X[5$, Theorem 2.4].

Behrouzi and Mahyar in [1] studied endomorphisms of some uniform subalgebras of $A(X)$ and some Banach function subalgebras of $\operatorname{Lip}_{A}(X, \alpha)$ and investigated some necessary and sufficient conditions for these endomorphisms to be compact, where $X$ is a compact plane set and $\alpha \in(0,1]$.

In Section 2, we study unital homomorphisms from natural Banach function subalgebras of $\operatorname{Lip}_{A}\left(X_{1}, K_{1}, \alpha_{1}\right)$ to natural Banach function subalgebras of $\operatorname{Lip}_{A}\left(X_{2}, K_{2}, \alpha_{2}\right)$ and investigate necessary and sufficient conditions for which these homomorphisms are compact. In Section 3, we determine the spectrum of unital compact endomorphisms of $\operatorname{Lip}_{A}(X, K, \alpha)$.

\section{Unital Compact Homomorphisms}

We first give a sufficient condition for which a continuous map $\varphi: X_{2} \rightarrow X_{1}$ induces a unital homomorphism $T$ from a subalgebra $B_{1}$ of $A\left(X_{1}, K_{1}\right)$ into a subalgebra $B_{2}$ of $A\left(X_{2}, K_{2}\right)$.

Proposition 2.1. Let $X_{j}$ and $K_{j}$ be compact plane sets with $\operatorname{int}\left(K_{j}\right) \neq \emptyset$ and $K_{j} \subseteq X_{j}$, and let $B_{j}$ be a subalgebra of $A\left(X_{j}, K_{j}\right)$ which is a natural Banach function algebra on $X_{j}$ under an algebra norm $\|\cdot\|_{j}$, where $j \in\{1,2\}$. If $\varphi \in B_{2}$ with $\varphi\left(X_{2}\right) \subseteq \operatorname{int}\left(K_{1}\right)$, then $\varphi$ induces a unital homomorphism $T: B_{1} \rightarrow B_{2}$. Moreover, if $Z \in B_{1}$, then $\varphi=T Z$.

Proof. The naturality of Banach function algebra $B_{2}$ on $X_{2}$ implies that $\sigma_{B_{2}}(h)=h\left(X_{2}\right)$, where $\sigma_{A}(h)$ is the spectrum of $h \in A$ in the Banach algebra $A$. Let $f \in B_{1}$. Since $\varphi \in B_{2}, \varphi\left(X_{2}\right) \subseteq$ $\operatorname{int}\left(K_{1}\right)$, and $f$ is analytic on $\operatorname{int}\left(K_{1}\right)$, we conclude that $f$ is analytic on an open neighborhood of $\sigma_{B_{2}}(\varphi)$. By using the Functional Calculus Theorem [2, Theorem 5.1 in Chapter I], there exists $g \in B_{2}$ such that $\hat{g}=f \circ \widehat{\varphi}$ on $\mathcal{M}\left(B_{2}\right)$. It follows that

$$
\begin{aligned}
g(z) & =e_{z}(g)=\widehat{g}\left(e_{z}\right)=f\left(\widehat{\varphi}\left(e_{z}\right)\right) \\
& =f\left(e_{z}(\varphi)\right)=f(\varphi(z))=(f \circ \varphi)(z),
\end{aligned}
$$

for all $z \in X_{2}$ and so $g=f \circ \varphi$. Therefore, $f \circ \varphi \in B_{2}$. This implies that the map $T: B_{1} \rightarrow B_{2}$ defined by $T f=f \circ \varphi$ is a unital homomorphism from $B_{1}$ into $B_{2}$, which is induced by $\varphi$. Now let $Z \in B_{1}$. Then $\varphi=T Z$ by Proposition 1.2. 
Corollary 2.2. Let $X$ and $K$ be compact plane sets with $\operatorname{int}(K) \neq \emptyset$ and $K \subseteq X$. Let $B$ be a subalgebra of $A(X, K)$ which is a natural Banach function algebra on $X$ under an algebra norm $\|\cdot\|_{B}$. If $\varphi \in B$ with $\varphi(X) \subseteq \operatorname{int}(K)$, then $\varphi$ induces a unital endomorphism $T$ of $B$. Moreover, if $Z \in B$, then $\varphi=T Z$.

Proposition 2.3. Suppose that $\alpha_{j} \in(0,1], z_{j} \in \mathbb{C}, 0<r_{j}<R_{j}, G_{j}=\mathbb{D}\left(z_{j}, R_{j}\right), \Omega_{j}=\mathbb{D}\left(z_{j}, r_{j}\right)$, $X_{j}=\overline{G_{j}}$, and $K_{j}=\overline{\Omega_{j}}$, where $j \in\{1,2\}$. Then for each $\rho \in\left(r_{1}, R_{1}\right]$ there exists a continuous map $\varphi_{\rho}: X_{2} \rightarrow X_{1}$ with $\varphi_{\rho}\left(X_{2}\right)=\overline{\mathbb{D}\left(z_{1}, \rho\right)}$ such that $\varphi_{\rho} \in \operatorname{Lip}_{A}\left(X_{2}, K_{2}, \alpha_{2}\right)$ and $\varphi_{\rho}$ does not induce any homomorphism from $\operatorname{Lip}_{A}\left(X_{1}, K_{1}, \alpha_{1}\right)$ to $\operatorname{Lip}_{A}\left(X_{2}, K_{2}, \alpha_{2}\right)$.

Proof. Let $\rho \in\left(r_{1}, R_{1}\right]$. We define the map $\varphi_{\rho}: X_{2} \rightarrow X_{1}$ by

$$
\varphi_{\rho}(z)= \begin{cases}z_{1}+\frac{\rho\left(z-z_{2}\right)}{r_{2}} & \left|z-z_{2}\right| \leq r_{2} \\ z_{1}+\frac{\rho\left(z-z_{1}\right)}{\left|z-z_{2}\right|} & r_{2}<\left|z-z_{2}\right| \leq R_{2} .\end{cases}
$$

Clearly, $\varphi_{\rho}$ is a continuous mapping, $\varphi_{\rho}\left(X_{2}\right)=\overline{\mathbb{D}\left(z_{1}, \rho\right)}$, and $\varphi_{\rho} \in \operatorname{Lip}_{A}\left(X_{2}, K_{2}, \alpha_{2}\right)$. We now define the function $f_{\rho}: X_{1} \rightarrow \mathbb{C}$ by

$$
f_{\rho}(z)=\left\{\begin{array}{l}
\frac{\rho\left(z-z_{1}\right)}{r_{1}}\left|z-z_{1}\right| \leq r_{1} \\
\frac{\rho\left(z-z_{1}\right)}{\left|z-z_{1}\right|} \quad r_{1}<\left|z-z_{1}\right| \leq R_{1}
\end{array}\right.
$$

Then, $f_{\rho} \in \operatorname{Lip}_{A}\left(X_{1}, K_{1}, \alpha_{1}\right)$. Since $0<r_{1} r_{2} / \rho<r_{2}$ and

$$
\left(f_{\rho} \circ \varphi_{\rho}\right)(z)= \begin{cases}\frac{\rho^{2}}{r_{1} r_{2}}\left(z-z_{2}\right) & \left|z-z_{2}\right| \leq \frac{r_{1} r_{2}}{\rho} \\ \frac{\rho\left(z-z_{2}\right)}{\left|z-z_{2}\right|} & \frac{r_{1} r_{2}}{\rho}<\left|z-z_{2}\right| \leq R_{2}\end{cases}
$$

we conclude that $f_{\rho} \circ \varphi_{\rho} \notin \operatorname{Lip}_{A}\left(X_{2}, K_{2}, \alpha_{2}\right)$. Therefore, $\varphi_{\rho}$ does not induce any homomorphism from $\operatorname{Lip}_{A}\left(X_{1}, K_{1}, \alpha_{1}\right)$ to $\operatorname{Lip}_{A}\left(X_{2}, K_{2}, \alpha_{2}\right)$. Hence, the proof is complete.

Corollary 2.4. Suppose that $\alpha \in(0,1], \lambda \in \mathbb{C}, 0<r<R, G=\mathbb{D}(\lambda, R), \Omega=\mathbb{D}(\lambda, r), X=\bar{G}$, and $K=\bar{\Omega}$. Then for each $\rho \in(r, R]$, there exists a continuous self-map $\varphi_{\rho}$ of $X$ with $\varphi_{\rho}(X)=\overline{\mathbb{D}(\lambda, \rho)}$ such that $\varphi_{\rho} \in \operatorname{Lip}_{A}(X, K, \alpha)$ and $\varphi_{\rho}$ does not induce any endomorphism of $\operatorname{Lip}_{A}(X, K, \alpha)$.

We now give a sufficient condition for a unital homomorphism from a subalgebra $B_{1}$ of $\operatorname{Lip}_{A}\left(X_{1}, K_{1}, \alpha_{1}\right)$ into a subalgebra $B_{2}$ of $\operatorname{Lip}_{A}\left(X_{2}, K_{2}, \alpha_{2}\right)$ to be compact.

Theorem 2.5. Suppose that $\alpha_{j} \in(0,1], X_{j}$ and $K_{j}$ are compact plane sets with $\operatorname{int}\left(K_{j}\right) \neq \emptyset$ and $K_{j} \subseteq X_{j}$, and $B_{j}$ is a subalgebra of $\operatorname{Lip}_{A}\left(X_{j}, K_{j}, \alpha_{j}\right)$ which is a natural Banach function algebra on $X_{j}$ under the norm $\|\cdot\|_{L i p\left(X_{j}, K_{j}, \alpha_{j}\right)}$, where $j \in\{1,2\}$. Let $\varphi: X_{2} \rightarrow X_{1}$ be a continuous mapping. If $\varphi$ is constant or $\varphi \in B_{2}$ with $\varphi\left(X_{2}\right) \subseteq \operatorname{int}\left(K_{1}\right)$, then $\varphi$ induces a unital compact homomorphism $T: B_{1} \rightarrow B_{2}$. 
Proof. If $\varphi: X_{2} \rightarrow X_{1}$ is constant, then the map $T: B_{1} \rightarrow B_{2}$ defined by $T f=f \circ \varphi$ is a unital homomorphism from $B_{1}$ into $B_{2}$ with $\operatorname{dim} T\left(B_{1}\right) \leq 1$, and so it is compact.

Let $\varphi: X_{2} \rightarrow X_{1}$ be a nonconstant mapping with $\varphi \in B_{2}$ and $\varphi\left(X_{2}\right) \subseteq \Omega_{1}$. Then the map $T: B_{1} \rightarrow B_{2}$ defined by $T f=f \circ \varphi$ is a unital homomorphism from $B_{1}$ to $B_{2}$ by Proposition 2.1. To prove the compactness of $T$, let $\left\{f_{n}\right\}_{n=1}^{\infty}$ be a bounded sequence in $B_{1}$ with $\left\|f_{n}\right\|_{\operatorname{Lip}\left(X_{1}, K_{1}, \alpha_{1}\right)} \leq 1$ for all $n \in \mathbb{N}$. This implies that $\left\{\left.f_{n}\right|_{K_{1}}\right\}_{n=1}^{\infty}$ is a bounded sequence in $C\left(K_{1}\right)$ which is equicontinuous on $\left(K_{1}, d_{1}^{\alpha_{1}}\right)$. By Arzela-Ascoli's theorem, $\left\{f_{n}\right\}_{n=1}^{\infty}$ has a subsequence $\left\{f_{n_{j}}\right\}_{j=1}^{\infty}$ such that $\left\{\left.f_{n_{j}}\right|_{K_{1}}\right\}_{j=1}^{\infty}$ is convergent in $C\left(K_{1}\right)$. Since $\left.f_{n_{j}}\right|_{K_{1}} \in A\left(K_{1}\right)$ for all $j \in \mathbb{N},\left\{\left.f_{n_{j}}\right|_{K_{1}}\right\}_{j=1}^{\infty}$ is convergent in $A\left(K_{1}\right)$. By Montel's theorem, the sequences $\left\{f_{n_{j}}\right\}_{j=1}^{\infty}$ and $\left\{f_{n_{j}}^{\prime}\right\}_{j=1}^{\infty}$ are uniformly convergent on the compact subsets of $\operatorname{int}\left(K_{1}\right)$. Since $\varphi\left(X_{2}\right)$ and $K_{1}$ are compact sets in the complex plane and $\varphi\left(X_{2}\right) \subseteq \operatorname{int}\left(K_{1}\right)$, by using Lemma 1.5, we deduce that there exists a finite union of uniformly regular sets in $\operatorname{int}\left(K_{1}\right)$ containing $\varphi\left(X_{2}\right)$, namely $Y$, and then a positive constant $C$ such that for every analytic complex-valued function $f$ on $\operatorname{int}\left(K_{1}\right)$ and any $z, w \in \varphi\left(X_{2}\right)$

$$
|f(z)-f(w)| \leq C|z-w|\left(\|f\|_{Y}+\left\|f^{\prime}\right\|_{Y}\right) .
$$

Therefore, there exists a positive constant $C$ such that

$$
\left|f_{n_{j}}(\varphi(z))-f_{n_{j}}(\varphi(w))\right| \leq C|\varphi(z)-\varphi(w)|\left(\left\|f_{n_{j}}\right\|_{Y}+\left\|f_{n_{j}}^{\prime}\right\|_{Y}\right)
$$

for all $j \in \mathbb{N}$ and any $z, w \in X_{2}$. Let $j, k \in \mathbb{N}$. Then, for all $z, w \in K_{2}$ with $\varphi(z) \neq \varphi(w)$, we have

$$
\begin{aligned}
& \frac{\left|\left(\left(f_{n_{j}} \circ \varphi\right)-\left(f_{n_{k}} \circ \varphi\right)\right)(z)-\left(\left(f_{n_{j}} \circ \varphi\right)-\left(f_{n_{k}} \circ \varphi\right)\right)(w)\right|}{|z-w|^{\alpha_{2}}} \\
& \quad=\frac{\left|\left(f_{n_{j}}-f_{n_{k}}\right)(\varphi(z))-\left(f_{n_{j}}-f_{n_{k}}\right)(\varphi(w))\right|}{|\varphi(z)-\varphi(w)|} \cdot \frac{|\varphi(z)-\varphi(w)|}{|z-w|^{\alpha_{2}}} \\
& \quad \leq C p_{\alpha_{2}, K_{2}}(\varphi)\left(\left\|f_{n_{j}}-f_{n_{k}}\right\|_{Y}+\left\|f_{n_{j}}^{\prime}-f_{n_{k}}^{\prime}\right\|_{Y}\right) .
\end{aligned}
$$

The above inequality is certainly true for all $z, w \in K_{2}$ with $z \neq w$ and $\varphi(z)=\varphi(w)$. Therefore,

$$
p_{\alpha_{2}, K_{2}}\left(\left(f_{n_{j}} \circ \varphi\right)-\left(f_{n_{k}} \circ \varphi\right)\right) \leq C p_{\alpha_{2}, K_{2}}(\varphi)\left(\left\|f_{n_{i}}-f_{n_{j}}\right\|_{Y}+\left\|f_{n_{i}}^{\prime}-f_{n_{j}}^{\prime}\right\|_{Y}\right)
$$

and so

$$
\left\|\left(f_{n_{j}} \circ \varphi\right)-\left(f_{n_{k}} \circ \varphi\right)\right\|_{\operatorname{Lip}\left(X_{2}, K_{2}, \alpha_{2}\right)} \leq\left(1+C p_{\alpha_{2}, K_{2}}(\varphi)\right)\left(\left\|f_{n_{j}}-f_{n_{k}}\right\|_{Y}+\left\|f_{n_{j}}^{\prime}-f_{n_{k}}^{\prime}\right\|_{Y}\right) .
$$

Since $Y$ is a compact subset of $\operatorname{int}\left(K_{1}\right)$, we deduce that the sequences $\left\{f_{n_{j}}\right\}_{j=1}^{\infty}$ and $\left\{f_{n_{j}}^{\prime}\right\}_{j=1}^{\infty}$ are convergent uniformly on $Y$. Therefore, $\left\{f_{n_{j}} \circ \varphi\right\}_{j=1}^{\infty}$ is a Cauchy sequence on $\operatorname{Lip}\left(X_{2}, K_{2}, \alpha_{2}\right)$, that is $\left\{T f_{n_{j}}\right\}_{j=1}^{\infty}$ is convergent in $\operatorname{Lip}\left(X_{2}, K_{2}, \alpha_{2}\right)$. Hence, $T$ is compact. 
Corollary 2.6. Suppose that $\alpha \in(0,1], X$ and $K$ are compact plane sets with $\operatorname{int}(K) \neq \emptyset$, and $K \subseteq X$. Let $B$ be a subalgebra of $\operatorname{Lip}_{A}(X, K, \alpha)$ which is a natural Banach function algebra on $X$ with the norm $\|\cdot\|_{L i p\left(X_{2}, K_{2}, \alpha_{2}\right)}$, and let $\varphi$ be a self-map of $X$. If $\varphi$ is constant or $\varphi \in B$ with $\varphi(X) \subseteq \operatorname{int}(K)$, then $\varphi$ induces a unital compact endomorphism of $B$.

Definition 2.7.

(a) A sector in $\mathbb{D}\left(z_{0}, r\right)$ at a point $\omega \in \partial \mathbb{D}\left(z_{0}, r\right)$ is the region between two straight lines in $\mathbb{D}\left(z_{0}, r\right)$ that meet at $\omega$ and are symmetric about the radius to $\omega$.

(b) If $f$ is a complex-valued function on $\mathbb{D}\left(z_{0}, r\right)$ and $\omega \in \partial \mathbb{D}\left(z_{0}, r\right)$, then $\angle \lim _{z \rightarrow \omega} f(z)=L$ means that $f(z) \rightarrow L$ as $z \rightarrow \omega$ through any sector at $\omega$. When this happens, we say that $L$ is angular (or non-tangential) limit of $f$ at $\omega$.

(c) An analytic map $\varphi: \mathbb{D}\left(z_{0}, r\right) \rightarrow \mathbb{D}_{\rho}$ has an angular derivation at a point $\omega \in \partial \mathbb{D}_{r}\left(z_{0}, r\right)$ if for some $\eta \in \partial \mathbb{D}_{\rho}$

$$
\angle \lim _{z \rightarrow \omega} \frac{\eta-f(z)}{\omega-z}
$$

exists (finitely). We call the limit the angular derivative of $\varphi$ at $\omega$ and denote it by $\angle \varphi^{\prime}(\omega)$.

Lemma 2.8. Let $0<r \leq 1$, and let $\varphi: \mathbb{D}\left(z_{0}, r\right) \rightarrow \mathbb{D}_{\rho}$ be an analytic function and $\psi: \mathbb{D} \rightarrow \mathbb{D}$ defined by $\psi(z)=(1 / \rho) \varphi\left(z_{0}+r z\right)$. Then $\varphi$ has angular derivation at $\omega \in \partial \mathbb{D}\left(z_{0}, r\right)$ if and only if $\psi$ has angular derivation at $\left(\omega-z_{0}\right) / r \in \partial \mathbb{D}$. Moreover,

$$
\angle \varphi^{\prime}(\omega)=\frac{r}{\rho} \angle \psi^{\prime}\left(\frac{\omega-z_{0}}{r}\right)
$$

The following result is a modification of Julia-Caratheodory's theorem. For further details and proof of Julia-Caratheodory's theorem, see [7, pages 295-300].

Theorem 2.9. Take $0<r \leq 1$. Let $\varphi: \mathbb{D}\left(z_{0}, r\right) \rightarrow \mathbb{D}$ be a nonconstant analytic function and $\omega \in \partial \mathbb{D}\left(z_{0}, r\right)$. Then the following are equivalent:

(i) $\liminf z \rightarrow \omega\left(\|\varphi\|_{\mathbb{D}_{r}}-|\varphi(z)|\right) /(r-|z|)=\delta<\infty$,

(ii) $\angle \lim _{z \rightarrow \omega}(\eta-\varphi(z)) /(\omega-z)$ exists for some $\eta \in \partial \mathbb{D}$,

(iii) $\angle \lim _{z \rightarrow \omega} \varphi^{\prime}(z)$ exists and $\angle \lim _{z \rightarrow \omega} \varphi(z)=\eta \in \partial \mathbb{D}$.

The boundary point $\eta$ in (ii) and (iii) is the same, and $\delta>0$ in (i). Also the limit of the difference quotients in (ii) coincides with the limit of the derivative in (iii), and both are equal to $\omega \bar{\eta} \delta$.

Note that the existence of the angular derivative $\varphi$ at $\omega \in \partial \mathbb{D}\left(z_{0}, r\right)$, according to Theorem 2.9, is equivalent to $\liminf _{z \rightarrow \omega}\left(\|\varphi\|_{\mathbb{D}\left(z_{0}, r\right)}-|\varphi(z)|\right) /\left(r-\left|z-z_{0}\right|\right)<\infty$. In this case the angular derivative of $\varphi$ at $\omega$ is nonzero.

Proposition 2.10. Let $X$ be a compact plane set, and let $\mathbb{D}\left(z_{0}, r\right) \subseteq X$ and $K=\overline{\mathbb{D}\left(z_{0}, r\right)}$. Suppose that $c \in \partial \mathbb{D}\left(z_{0}, r\right)$ and $\varphi \in \operatorname{Lip}_{A}(X, K, 1)$ is a nonconstant function such that $|\varphi(c)|=\|\varphi\|_{\overline{\mathbb{D}\left(z_{0}, r\right)}}$. Then the angular derivative of $\varphi$ at $c$ exists and is nonzero. 
Proof. Let $\Gamma=\left\{z \in \mathbb{D}\left(z_{0}, r\right):|z-c| /\left(r-\left|z-z_{0}\right|\right)<2\right\}$. For every $z \in \Gamma$ we have

$$
\frac{\|\varphi\|_{\mathbb{D}\left(z_{0}, r\right)}-|\varphi(z)|}{r-\left|z-z_{0}\right|}=\frac{|\varphi(c)|-|\varphi(z)|}{r-\left|z-z_{0}\right|} \leq \frac{|z-c|}{r-\left|z-z_{0}\right|} \frac{|\varphi(z)-\varphi(c)|}{|z-c|}<2 p_{1, K}(\varphi) .
$$

Therefore, $\liminf _{z \rightarrow \omega}\left(\|\varphi\|_{\mathbb{D}\left(z_{0}, r\right)}-|\varphi(z)|\right) /\left(r-\left|z-z_{0}\right|\right)<\infty$, and, by Theorem 2.9, the proof is complete.

\section{Definition 2.11.}

(a) A plane set $X$ at $c \in \partial X$ has an internal circular tangent if there exists a disc $D$ in the complex plane such that $c \in \partial D$ and $\bar{D} \backslash\{c\} \subseteq \operatorname{int}(X)$.

(b) A plane set $X$ is called strongly accessible from the interior if it has an internal circular tangent at each point of its boundary. Such sets include the closed unit disc $\overline{\mathbb{D}}$ and $\overline{\mathbb{D}\left(z_{0}, r\right)} \backslash \bigcup_{k=1}^{n} \mathbb{D}\left(z_{k}, r_{k}\right)$, where closed discs $\overline{\mathbb{D}\left(z_{k}, r_{k}\right)}$ are mutually disjoint in $\mathbb{D}\left(z_{0}, r\right)$.

(c) A compact plane set $X$ has peak boundary with respect to $B \subseteq C(X)$ if for each $c \in \partial X$ there exists a nonconstant function $h \in B$ such that $\|h\|_{X}=h(c)=1$.

Example 2.12. The closed unit disc $\overline{\mathbb{D}}$ has peak boundary with respect to $A(\overline{\mathbb{D}})$ because, if $c \in \partial \overline{\mathbb{D}}$, then the function $h: \overline{\mathbb{D}} \rightarrow C$ defined by $h(z)=(1 / 2)(1+\bar{c} z)$ belongs to $A(\overline{\mathbb{D}})$ and satisfies $\|h\|_{\bar{D}}=h(c)=1$.

Let $X$ be a compact plane set. The algebra $R(X)$ consists of all functions in $C(X)$ which can be approximated by rational functions with poles off $X$. It is known that $R(X)$ is a natural uniform algebra on $X$.

Example 2.13. Let $X$ be a compact plane set such that $\mathbb{C} \backslash X$ is strongly accessible from the interior. If $R(X) \subseteq B \subseteq C(X)$, then $X$ has a peak boundary with respect to $B$.

Proof. Let $z_{0} \in \mathbb{C} \backslash X$. Since $\mathbb{C} \backslash X$ is strongly accessible from the interior, for each $c \in \partial(\mathbb{C} \backslash X)$, there exists a $\delta>0$ such that $\left|c-z_{0}\right|=\delta$ and $\overline{\mathbb{D}\left(z_{0}, \delta\right)} \subseteq \operatorname{int}(\mathbb{C} \backslash X)$. Now, we define the function $h: X \rightarrow \mathbb{C}$ by

$$
h(z)=\frac{\delta^{2}}{\left(\bar{c}-\overline{z_{0}}\right)\left(z-z_{0}\right)} .
$$

Then $h \in B,\|h\|_{X}=h(c)=1$.

Theorem 2.14. Let $X_{1}$ be a compact plane set such that $G_{1}=\operatorname{int}\left(X_{1}\right)$ is connected, and $\overline{G_{1}}=X_{1}$. Suppose that $X_{1}$ has peak boundary with respect to $\operatorname{Lip}_{A}\left(X_{1}, 1\right)$. Let $\Omega_{1} \subseteq G_{1}$ be a bounded connected open set in the complex plane, and let $K_{1}=\overline{\Omega_{1}}$. Let $\Omega_{2}$ be a bounded connected open set in the complex plane, and let $K_{2}=\overline{\Omega_{2}}$ such that $K_{2}$ is strongly accessible from the interior. Suppose that $X_{2}$ is a compact plane set such that $K_{2} \subseteq X_{2}$. If $T: \operatorname{Lip}_{A}\left(X_{1}, K_{1}, 1\right) \rightarrow \operatorname{Lip}_{A}\left(X_{2}, K_{2}, 1\right)$ is a unital compact homomorphism, then $T$ is induced by a continuous mapping $\varphi: X_{2} \rightarrow X_{1}$ such that $\varphi$ is constant on $K_{2}$ or $\varphi\left(K_{2}\right) \subseteq G_{1}=\operatorname{int}\left(X_{1}\right)$. Moreover, $\varphi=T Z$. 
Proof. Since $\operatorname{Lip}_{A}\left(X_{1}, K_{1}, 1\right)$ and $\operatorname{Lip}_{A}\left(X_{2}, K_{2}, 1\right)$ are, respectively, natural Banach function algebras on $X_{1}$ and $X_{2}, T: \operatorname{Lip}_{A}\left(X_{1}, K_{1}, 1\right) \rightarrow \operatorname{Lip}_{A}\left(X_{2}, K_{2}, 1\right)$ is a unital homomorphism, $X_{1}$ is a compact plane set, and $Z \in \operatorname{Lip}_{A}\left(X_{1}, K_{1}, 1\right)$, we conclude that $T$ is induced by $\varphi=T Z$ and so $\varphi \in \operatorname{Lip}_{A}\left(X_{2}, K_{2}, 1\right)$ by Proposition 1.2. Suppose that $\varphi$ is nonconstant on $\Omega_{2}$. Since $\varphi$ is analytic on $\Omega_{2}$, we deduce that $\varphi\left(\Omega_{2}\right)$ is an open subset of $X_{1}$ and so $\varphi\left(\Omega_{2}\right) \subseteq G_{1}$. We now show that $\varphi\left(K_{2}\right) \subseteq G_{1}$. Suppose that $\varphi\left(K_{2}\right) \nsubseteq G_{1}$. Then there exists $c \in \partial K_{2}$ such that $\varphi(c) \in \partial X_{1}$. Since $X_{1}$ has peak boundary with respect to $\operatorname{Lip}_{A}\left(X_{1}, 1\right)$, there exists a nonconstant function $h \in \operatorname{Lip}_{A}\left(X_{1}, 1\right)$ such that $\|h\|_{X_{1}}=h(\varphi(c))=1$. We now define the sequence $\left\{f_{n}\right\}_{n=1}^{\infty}$ of complex-valued functions on $X_{1}$ by $f_{n}(z)=(1 / n) h^{n}(z)$. Let $n \in \mathbb{N}$. Then

$$
\begin{aligned}
& \left\|f_{n}\right\|_{X_{1}}=\frac{1}{n}\left(\|h\|_{X_{1}}\right)^{n}=\frac{1}{n} \\
p_{1, K_{1}}\left(f_{n}\right)= & \sup \left\{\frac{\left|h^{n}(z)-h^{n}(w)\right|}{n|z-w|}: z, w \in K_{1}, z \neq w\right\} \\
\leq & \sup \left\{\frac{|h(z)-h(w)|}{|z-w|}: z, w \in K_{1}, z \neq w\right\} \\
\leq & \sup \left\{\frac{|h(z)-h(w)|}{|z-w|}: z, w \in X_{1}, z \neq w\right\} \\
\leq & p_{1, X_{1}}(h) .
\end{aligned}
$$

Thus

$$
\left\|f_{n}\right\|_{\operatorname{Lip}\left(X_{1}, K_{1}, 1\right)} \leq \frac{1}{n}+p_{1, X_{1}}(h) \leq 1+p_{1, X_{1}}(h),
$$

by (2.14) and (2.15). This implies that $\left\{f_{n}\right\}_{n=1}^{\infty}$ is a bounded sequence in $\operatorname{Lip}_{A}\left(X_{1}, K_{1}, 1\right)$. The compactness of homomorphism $T$ implies that there exists a subsequence $\left\{f_{n_{j}}\right\}_{j=1}^{\infty}$ of $\left\{f_{n}\right\}_{n=1}^{\infty}$ and a function $g$ in $\operatorname{Lip}_{A}\left(X_{2}, K_{2}, 1\right)$ such that

$$
\lim _{j \rightarrow \infty}\left\|T f_{n_{j}}-g\right\|_{\operatorname{Lip}\left(X_{2}, K_{2}, 1\right)}=0
$$

This implies that

$$
\lim _{j \rightarrow \infty}\left\|T f_{n_{j}}-g\right\|_{X_{2}}=0
$$

On the other hand, we have $\left\|T f_{n_{j}}\right\|_{X_{2}} \leq 1 / n_{j}$ for all $j \in \mathbb{N}$ by (2.14). Hence,

$$
\lim _{j \rightarrow \infty}\left\|T f_{n_{j}}\right\|_{X_{2}}=0
$$

By (2.18) and (2.19), $g=0$. Therefore, by (2.17) we have

$$
\lim _{j \rightarrow \infty}\left\|T f_{n_{j}}\right\|_{\operatorname{Lip}\left(X_{2}, K_{2}, 1\right)}=0 .
$$


This implies that

$$
\lim _{j \rightarrow \infty} p_{1, K_{2}}\left(f_{n_{j}} \circ \varphi\right)=0
$$

Assume that $\varepsilon>0$. By (2.21), there exists a natural number $N$ such that for each $j \in \mathbb{N}$ with $j \geq N$

$$
\sup \left\{\frac{\left|\left(f_{n_{j}} \circ \varphi\right)(z)-\left(f_{n_{j}} \circ \varphi\right)(w)\right|}{|z-w|}: z, w \in K_{2}, z \neq w\right\}<\varepsilon
$$

In particular,

$$
\sup \left\{\frac{\left|((h \circ \varphi)(z))^{n_{N}}-((h \circ \varphi)(w))^{n_{N}}\right|}{n_{N}|z-w|}: z, w \in K_{2}, z \neq w\right\}<\varepsilon .
$$

This implies that

$$
\frac{1}{n_{N}} \sup \left\{\frac{\left|((h \circ \varphi)(z))^{n_{N}}-((h \circ \varphi)(c))^{n_{N}}\right|}{|z-c|}: z \in K_{2}, z \neq c\right\}<\varepsilon
$$

Since $c \in \partial K_{2}$ and $K_{2}$ is strongly accessible from the interior, there exists an open disc $D=$ $\mathbb{D}\left(z_{0}, r\right)$ such that $c \in \partial D$ and $\bar{D} \backslash\{c\} \subseteq \operatorname{int}\left(K_{2}\right)$. Since $\varphi$ is analytic on $\operatorname{int}(\bar{D}) \subseteq \operatorname{int}\left(K_{2}\right)$ and $h$ is analytic on $\varphi(\bar{D}) \subseteq \operatorname{int}\left(X_{1}\right)$, we deduce that $h \circ \varphi$ is analytic on int $(\bar{D})$. On the other hand, we can easily show that

$$
p_{1, \bar{D}}(h \circ \varphi) \leq p_{1, X_{1}}(h) p_{1, K_{2}}(\varphi)<\infty .
$$

Therefore, $h \circ \varphi \in \operatorname{Lip}_{A}\left(X_{2}, \bar{D}, 1\right)$. Since $\|h\|_{X_{1}}=h(\varphi(c))=1$, we conclude that

$$
(h \circ \varphi)(c)=\|h \circ \varphi\|_{\bar{D}}=1 .
$$

We claim that $h \circ \varphi$ is constant on $D$. If $h \circ \varphi$ is nonconstant on $D$, then, by Proposition 2.10, $\angle(h \circ \varphi)^{\prime}(c)$ exists and is nonzero and since $(h \circ \varphi)^{n_{N}}(c)=1,(h \circ \varphi)^{n_{N}}(c) \in \partial D$. If $\Gamma$ is a sector in $D$ at $c \in \partial D$, then

$$
\frac{1}{n_{N}} \angle \lim _{z \rightarrow \Gamma}\left|\frac{(h \circ \varphi)^{n_{N}}(z)-(h \circ \varphi)^{n_{N}}(c)}{z-c}\right| \leq \varepsilon
$$

by (2.24). Thus

$$
\frac{1}{n_{N}} \angle\left((h \circ \varphi)^{n_{N}}\right)^{\prime}(c) \leq \varepsilon
$$


But

$$
\angle\left((h \circ \varphi)^{n_{N}}\right)^{\prime}(c)=n_{N}(h \circ \varphi)^{n_{N}-1}(c) \cdot \angle(h \circ \varphi)^{\prime}(c) .
$$

Hence, by (2.28) we have

$$
\angle(h \circ \varphi)^{\prime}(c)=\frac{1}{n_{N}} \angle\left((h \circ \varphi)^{n_{N}}\right)^{\prime}(c) \leq \varepsilon .
$$

Since $\varepsilon$ is assumed to be a positive number, we conclude that $\angle(h \circ \varphi)^{\prime}(c)=0$, contradicting to $\angle(h \circ \varphi)^{\prime}(c) \neq 0$. Hence, our claim is justified. Since $\varphi$ is nonconstant on $K_{2}, \varphi$ is a nonconstant analytic function on connected open set $D$. This implies that $\varphi(D)$ is a connected open set in the complex plane. This implies that $h$ is constant on connected open set $G_{1}$. The continuity of $h$ on $X_{1}=\overline{G_{1}}$ follows that $h$ is constant on $\overline{G_{1}}=X_{1}$. This contradicting to $h$ is nonconstant on $X_{1}$. Therefore, $\varphi\left(K_{2}\right) \subseteq G_{1}$.

Corollary 2.15. Let $X$ be a compact plane set such that $G=\operatorname{int}(X)$ is connected and $\bar{G}=X$. Let $\Omega \subseteq G$ be a bounded connected open set in the complex plane, and let $K=\bar{\Omega}$. Suppose that $K$ is strongly accessible from the interior and $X$ has peak boundary with respect to $\operatorname{Lip}_{A}(X, 1)$. If $T$ is a unital compact endomorphism of $\operatorname{Lip}_{A}(X, K, 1)$, then $T$ is induced by a continuous self-map $\varphi$ of $X$ such that $\varphi$ is constant on $K$ or $\varphi(K) \subseteq G=\operatorname{int}(X)$. Moreover, $\varphi=T Z$.

Lemma 2.16. Let $G$ and $\Omega$ be bounded connected open sets in the complex plane with $\Omega \subseteq G$, and let $X=\bar{G}$ and $K=\bar{\Omega}$. Then for each $c \in G \backslash K$ there exists a function $f_{c} \in \operatorname{Lip}{ }_{A}(X, K, 1)$ such that $f_{c}$ is not analytic at $c$.

Proof. Let $c \in G \backslash K$. Then there is a positive number $r$ such that

$$
\{z \in \mathbb{C}:|z-c| \leq r\} \subseteq G \backslash K
$$

We now define the function $f_{c}: X \rightarrow \mathbb{C}$ by

$$
f_{c}(z)= \begin{cases}z-c & z \in X,|z-c| \geq r, \\ \frac{(1+r)(z-c)}{1+|z-c|} & z \in X,|z-c|<r .\end{cases}
$$

It is easily seen that $f_{c} \in \operatorname{Lip}_{A}(X, K, 1)$ and $f_{c}$ is not analytic at $c$.

Definition 2.17. Let $X$ and $K$ be compact plane sets such that $K \subseteq X$. We say that $K$ has peak $K$-boundary with respect to $B \subseteq A(X, K)$ if for each $c \in \partial K$ there is a function $h \in B$ such that $h$ is nonconstant on $K$ and $\|h\|_{X}=h(c)=1$.

Example 2.18. Let $r \in(0,1]$ and $K=\overline{\mathbb{D}_{r}}$. Suppose that $\operatorname{Lip}_{A}(\overline{\mathbb{D}}, K, 1) \subseteq B \subseteq A(\overline{\mathbb{D}}, K)$. Then $K$ has peak $K$-boundary with respect to $B$.

Proof. We first assume that $r=1$. If for each $c \in \partial \overline{\mathbb{D}}$ the function $h: \overline{\mathbb{D}} \rightarrow \mathbb{C}$ defined by $h(z)=(1 / 2)(1+\bar{c} z)$, then $h \in B, h$ is nonconstant on $K=\overline{\mathbb{D}}$ and $h(c)=1=\|h\|_{\overline{\mathbb{D}}}$. 
We now assume that $0<r<1$. For each $c \in \partial K$, set $z_{0}=(1+r) c / r$. Then $z_{0} \in \mathbb{C} \backslash \overline{\mathbb{D}}$. Define the function $h: \overline{\mathbb{D}} \rightarrow \mathbb{C}$ by

$$
h(z)= \begin{cases}-\frac{r}{c\left(z-z_{0}\right)} & z \in \overline{\mathbb{D}},\left|z-z_{0}\right| \geq 1 \\ -\frac{r\left|z-z_{0}\right|}{\bar{c}\left(z-z_{0}\right)} & z \in \overline{\mathbb{D}},\left|z-z_{0}\right|<1 .\end{cases}
$$

It is easily seen that $h \in \operatorname{Lip}_{A}(\overline{\mathbb{D}}, K, 1)$ and $\|h\|_{\overline{\mathbb{D}}}=1=h(c)$.

Lemma 2.19. Let $\Omega$ be a connected open set in the complex plane, and let $\varphi$ be a one-to-one analytic function on $\Omega$. If $f$ is a continuous complex-valued function on $\varphi(\Omega)$ and $f \circ \varphi$ is analytic on $\Omega$, then $f$ is an analytic function on $\varphi(\Omega)$.

Proof. By [8, Theorem 7.5 and Corollary 7.6 in Chapter IV], we deduce that $\varphi(\Omega)$ is a connected open set in the complex plane, $\varphi^{\prime}(z) \neq 0$ for all $z \in \Omega$, and $\varphi^{-1}: \varphi(\Omega) \rightarrow \Omega$ is an analytic function on $\varphi(\Omega)$. Since $f=f \circ \varphi \circ \varphi^{-1}$ on $\varphi(\Omega)$, we conclude that $f$ is analytic on $\varphi(\Omega)$.

Theorem 2.20. Let $X_{1}$ be a compact plane set such that $G_{1}=\operatorname{int}\left(X_{1}\right)$ is connected and $\overline{G_{1}}=X_{1}$. Suppose that $K_{1}$ is a compact subset of $X_{1}$ such that $\Omega_{1}=\operatorname{int}\left(K_{1}\right)$ is connected, $K_{1}=\overline{\Omega_{1}}$, and $K_{1}$ has peak $K_{1}$-boundary with respect to $\operatorname{Lip}_{A}\left(X_{1}, K_{1}, 1\right)$. Let $\Omega_{2}$ be a bounded connected open set in the complex plane, and let $K_{2}=\overline{\Omega_{2}}$ such that $K_{2}$ is strongly accessible from the interior. Suppose that $X_{2}$ is a compact plane set such that $K_{2} \subseteq X_{2}$. If $T: \operatorname{Lip}_{A}\left(X_{1}, K_{1}, 1\right) \rightarrow \operatorname{Lip}_{A}\left(X_{2}, K_{2}, 1\right)$ is a unital compact homomorphism and $T Z$ is one-to-one on $\Omega_{2}$, then $T$ is induced by a continuous mapping $\varphi: X_{2} \rightarrow X_{1}$ such that $\varphi=T Z$ and $\varphi\left(K_{2}\right) \subseteq \Omega_{1}=\operatorname{int}\left(K_{1}\right)$.

Proof. Since $\operatorname{Lip}_{A}\left(X_{1}, K_{1}, 1\right)$ and $\operatorname{Lip}_{A}\left(X_{2}, K_{2}, 1\right)$ are, respectively, natural Banach function algebras on $X_{1}$ and $X_{2}, T: \operatorname{Lip}_{A}\left(X_{1}, K_{1}, 1\right) \rightarrow \operatorname{Lip}_{A}\left(X_{2}, K_{2}, 1\right)$ is a unital homomorphism, $X_{1}$ is a compact plane set, and $Z \in \operatorname{Lip}_{A}\left(X_{1}, K_{1}, 1\right)$, we conclude that $T$ is induced by $\varphi=T Z$ and so $\varphi \in \operatorname{Lip}_{A}\left(X_{2}, K_{2}, 1\right)$ by Proposition 1.2.

To prove $\varphi\left(K_{2}\right) \subseteq \Omega_{1}$, we first show that $\varphi\left(\Omega_{2}\right) \subseteq K_{1}$. Since $\varphi$ is a one-to-one analytic mapping on $\Omega_{2}$, we conclude that $\varphi\left(\Omega_{2}\right)$ is an open set in the complex plane. This follows that $\varphi\left(\Omega_{2}\right) \subseteq \operatorname{int}\left(X_{1}\right)=G_{1}$ since $\varphi\left(\Omega_{2}\right) \subseteq X_{1}$. Suppose that $\varphi\left(\Omega_{2}\right) \nsubseteq K_{1}$. Then there exists $\lambda \in \Omega_{2}$ such that $\varphi(\lambda) \in G_{1} \backslash K_{1}$. By Lemma 2.16, there exists a function $f \in \operatorname{Lip}_{A}\left(X_{1}, K_{1}, 1\right)$ such that $f$ is not analytic at $\varphi(\lambda)$. But $f \circ \varphi=T f \in \operatorname{Lip}_{A}\left(X_{2}, K_{2}, 1\right)$, so that $f \circ \varphi$ is analytic on $\Omega_{2}$. Since $f$ is continuous on $\varphi\left(\Omega_{2}\right)$ and $\varphi$ is a one-to-one analytic function on $\Omega_{2}$, we conclude that $f$ is analytic on $\varphi\left(\Omega_{2}\right)$ by Lemma 2.19. This contradicts to the fact $f$ is not analytic at $\varphi(\lambda) \in \varphi\left(\Omega_{2}\right)$. Therefore, $\varphi\left(\Omega_{2}\right) \subseteq K_{1}$ so $\varphi\left(\Omega_{2}\right) \subseteq \operatorname{int}\left(K_{1}\right)=\Omega_{1}$ since $\varphi\left(\Omega_{2}\right)$ is an open set in the complex plane. Since $\varphi$ is continuous on $K_{2}, \varphi\left(\Omega_{2}\right) \subseteq \Omega_{1}, K_{2}=\overline{\Omega_{2}}$, and $K_{1}=\overline{\Omega_{1}}$, we can easily show that $\varphi\left(K_{2}\right) \subseteq K_{1}$. We now show that $\varphi\left(K_{2}\right) \subseteq \Omega_{1}$. Suppose that $\varphi\left(K_{2}\right) \nsubseteq \Omega_{1}$. Then there exists $c \in \partial K_{2}$ such that $\varphi(c) \in \partial K_{1}$. Since $K_{1}$ has peak $K_{1}$-boundary with respect to $\operatorname{Lip}_{A}\left(X_{1}, K_{1}, 1\right)$, there exists a function $h \in \operatorname{Lip}_{A}\left(X_{1}, K_{1}, 1\right)$ such that $h$ is not constant on $K_{1}$ and

$$
\|h\|_{X_{1}}=h(\varphi(c))=1
$$


Applying the similar argument used in the proof of Theorem 2.14, we can prove that $h$ is constant on $K_{1}$. This contradiction shows that $\varphi\left(K_{2}\right) \subseteq \Omega_{1}$.

Corollary 2.21. Let $X$ be a compact plane set such that $G=\operatorname{int}(X)$ is connected and $\bar{G}=X$. Let $K$ be a compact subset of $X$ such that $\Omega=\operatorname{int}(K)$ is connected and $K=\bar{\Omega}$. Suppose that $K$ has peak $K$-boundary with respect to $\operatorname{Lip}_{A}(X, K, 1)$ and $K$ is strongly accessible from the interior. If $T$ is a unital compact endomorphism of $\operatorname{Lip}_{A}(X, K, 1)$ and $T Z$ is a one-to-one mapping on $\Omega$, then $T$ is induced by a continuous self-map $\varphi$ of $X$ such that $\varphi=T Z$ and $\varphi(K) \subseteq \Omega=\operatorname{int}(K)$.

\section{Spectrum of Unital Compact Endomorphisms}

In this section we determine the spectrum of a unital compact endomorphism of a subalgebra of $\operatorname{Lip}_{A}(X, K, \alpha)$ which is a natural Banach function algebra with the norm $\|\cdot\|_{\operatorname{Lip}(X, K, \alpha)}$.

The following result is a modification of [9, Theorem 1.7] for unital compact endomorphisms of natural Banach function algebras.

Theorem 3.1. Let $X$ be a compact Hausdorff space and $B$ a natural Banach function algebra on X. If T is a unital compact endomorphism of $B$ induced by a self-map $\varphi: X \rightarrow X$, then $\bigcap_{n=0}^{\infty} \varphi_{n}(X)$ is finite, and if $X$ is connected, $\bigcap_{n=0}^{\infty} \varphi_{n}(X)$ is singleton where $\varphi_{n}$ is the nth iterate of $\varphi$, that is, $\varphi_{0}(x)=x$ and $\varphi_{n}(x)=\varphi\left(\varphi_{n-1}(x)\right)$. If $\bigcap_{n=0}^{\infty} \varphi_{n}(X)=\left\{x_{0}\right\}$, then $x_{0}$ is a fixed point for $\varphi$. In fact, if $F=\bigcap_{n=0}^{\infty} \varphi_{n}(X)$, then $\varphi(F)=F$.

Theorem 3.2. Suppose that $X$ is a compact plane set with $\operatorname{int}(X) \neq \emptyset, \Omega$ is a connected open set in the complex plane with $\Omega \subseteq \operatorname{int}(X)$, and $K=\bar{\Omega}$. Let $B$ be a subalgebra of $A(X, K)$ containing the coordinate function $Z$ which is a natural Banach function algebra on $X$ with an algebra norm $\|\cdot\|_{B}$. Let $T$ be a unital compact endomorphism of $B$ induced by a self-map $\varphi$ of $X$. If $\varphi(X) \subseteq \operatorname{int}(K)$ and $z_{0}$ is a fixed point of $\varphi$, then

$$
\sigma(T)=\{0,1\} \cup\left\{\left(\varphi^{\prime}\left(z_{0}\right)\right)^{n}: n \in \mathbb{N}\right\}
$$

Proof. Clearly 0 and also $1 \in \sigma(T)$ since $T\left(1_{X}\right)=1_{X}$. If $\varphi$ is constant then the proof is complete. Let $\lambda \in \sigma(T) \backslash\{0,1\}$. The compactness of $T$ implies that there exists $f \in B \backslash\{0\}$ such that $T f=f \circ \varphi=\lambda f$. Since $\varphi\left(z_{0}\right)=z_{0} \in \operatorname{int}(K), f\left(z_{0}\right)=0$. We claim that $f^{(j)}\left(z_{0}\right) \neq 0$ for some $j \in \mathbb{N}$. If $f^{(n)}\left(z_{0}\right)=0$ for all $n \in \mathbb{N}$, then $f=0$ on an open disc with center $z_{0}$ and so on $\Omega$. By maximum modules principle, it follows that $f=0$ on $X$ since $\varphi(X) \subseteq \Omega, \lambda \in \mathbb{C} \backslash\{0\}$, and $\lambda f(z)=f(\varphi(z))$ for all $z \in X$. This contradicts to $f \neq 0$. Hence, our claim is justified. Let $m=\min \left\{n \in \mathbb{N}: f^{(n)}\left(z_{0}\right) \neq 0\right\}$. Then $f^{(k)}\left(z_{0}\right)=0$ for all $k \in\{0, \ldots, n-1\}$ and $f^{(m)}\left(z_{0}\right) \neq 0$. By $m$ times differentiation of $f \circ \varphi=\lambda f$, we have $\left(\varphi^{\prime}\left(z_{0}\right)\right)^{m} f^{(m)}\left(\varphi\left(z_{0}\right)\right)=\lambda f^{(m)}\left(z_{0}\right)$, and therefore $\lambda=\left(f^{\prime}\left(z_{0}\right)\right)^{m}$. Then $\sigma(T) \backslash\{0,1\} \subseteq\left\{\left(\varphi^{\prime}\left(z_{0}\right)\right)^{n}: n \in \mathbb{N}\right\}$.

Conversely, first we show that, if $\lambda \in \sigma(T)$ with $|\lambda|=1$, then $\lambda=1$. Let $\lambda \in \sigma(T)$ and $|\lambda|=1$. The compactness of $T$ implies that there exists $g \in B \backslash\{0\}$ such that $g \circ \varphi=\lambda g$. It follows that $|g \circ \varphi|=|g|$. Since $\varphi(K) \subseteq \operatorname{int}(K)=\Omega$ and $g$ is analytic on the connected open set $\Omega$, we conclude that $g$ is constant on $\Omega$ by maximum modules principle. Since $\varphi(X) \subseteq \Omega$, $g \circ \varphi=\lambda g$, and $\lambda \in \mathbb{C} \backslash\{0\}$, we deduce that $g$ is constant on $X$. Applying again $g \circ \varphi=\lambda g$ implies that $\lambda=1$ since $g \in B \backslash\{0\}$. 
We now claim that $\varphi^{\prime}\left(z_{0}\right) \in \sigma(T)$. If $\varphi^{\prime}\left(z_{0}\right) \notin \sigma(T)$, then there exists a nonzero linear operator $S: B \rightarrow B$ such that

$$
\left(T-\varphi^{\prime}\left(z_{0}\right) I\right) S=I
$$

Since $Z-z_{0} 1_{X} \in B, h=S\left(Z-z_{0} 1_{X}\right) \in B$ and so

$$
h \circ \varphi-\varphi^{\prime}\left(z_{0}\right) h=Z-z_{0} 1_{X}
$$

by (3.2). By differentiation at $z_{0}$, we have

$$
0=h^{\prime}\left(\varphi\left(z_{0}\right)\right) \varphi^{\prime}\left(z_{0}\right)-\varphi^{\prime}\left(z_{0}\right) h^{\prime}\left(z_{0}\right)=1 \text {, }
$$

this is a contradiction. Hence, our claim is justified.

We now show that $\left(\varphi^{\prime}\left(z_{0}\right)\right)^{n} \in \sigma(T)$ for all $n \in \mathbb{N}$. If $\varphi^{\prime}\left(z_{0}\right)=0$ or $\left|\varphi^{\prime}\left(z_{0}\right)\right|=1$, the proof is complete. Suppose that $\varphi^{\prime}\left(z_{0}\right) \neq 0$ and $\left|\varphi^{\prime}\left(z_{0}\right)\right| \neq 1$. If $\left(\varphi^{\prime}\left(z_{0}\right)\right)^{j} \notin \sigma(T)$ for some $j \in \mathbb{N}$ with $j>1$, then there exists a nonzero linear operator $S_{j}: B \rightarrow B$ such that

$$
\left(T-\left(\varphi^{\prime}\left(z_{0}\right)\right)^{j} I\right) S_{j}=I
$$

Since $\left(Z-z_{0} 1_{X}\right)^{j} \in B, h_{j}=S_{j}\left(Z-z_{0} 1_{X}\right)^{j} \in B$ and so

$$
h_{j} \circ \varphi-\left(\varphi^{\prime}\left(z_{0}\right)\right)^{j} h_{j}=\left(Z-z_{0} 1_{X}\right)^{j}
$$

by (3.5). By $j-1$ times differentiation at $z_{0}$, we have

$$
h_{j}\left(z_{0}\right)=h_{j}^{\prime}\left(z_{0}\right)=\cdots=h_{j}^{(j-1)}\left(z_{0}\right)=0,
$$

and by $j$ times differentiation at $z_{0}$, we have

$$
0=\left(\varphi^{\prime}\left(z_{0}\right)\right)^{j} h_{j}^{(j)}\left(\varphi\left(z_{0}\right)\right)-\left(\varphi^{\prime}\left(z_{0}\right)\right)^{j} h_{j}^{(j)}\left(z_{0}\right)=j !
$$

this is a contradiction. Thus, $\left(\varphi^{\prime}\left(z_{0}\right)\right)^{n} \in \sigma(T)$ for all $n \in \mathbb{N}$. This completes the proof.

Corollary 3.3. Let $B$ and $T$ satisfy the conditions of Theorem 3.2, and let $B$ be a natural Banach function algebra with the norm $\|\cdot\|_{\alpha, K}$. If $F$ is a finite set such that $\varphi(F)=F$, then there exist $z_{0} \in F$ and $m \in \mathbb{N}$ such that

$$
\left\{\lambda^{m}: \lambda \in \sigma(T)\right\}=\{0,1\} \cup\left\{\left(\varphi_{m}^{\prime}\left(z_{0}\right)\right)^{n}: n \in \mathbb{N}\right\}
$$

Proof. Since $F$ is a finite set and $\varphi(F)=F$, there exist $z_{0} \in F$ and $m \in \mathbb{N}$ such that $\varphi_{m}\left(z_{0}\right)=z_{0}$. Since $\varphi(X) \subseteq \operatorname{int}(K)$, so $z_{0} \in \operatorname{int}(K)$. If $\varphi$ is constant, then the proof is complete. When $\varphi$ is 
nonconstant, we define $\tilde{T}: B \rightarrow B$ by $\tilde{T} f=f \circ \varphi_{m}$. Then $\tilde{T}$ is a compact endomorphism of $B$ induced by $\varphi_{m}$ by Corollary 2.6 and $\varphi_{m}\left(z_{0}\right)=z_{0}$. Therefore,

$$
\sigma(\tilde{T})=\{0,1\} \cup\left\{\left(\varphi_{m}^{\prime}\left(z_{0}\right)\right)^{n}: n \in \mathbb{N}\right\}
$$

by Theorem 3.2. Since $T f=f \circ \varphi$ and $\tilde{T} f=f \circ \varphi_{m}$ for all $f \in B$, we have $\widetilde{T}=T^{m}$. By Spectral Mapping Theorem, $\sigma\left(T^{m}\right)=\left\{\lambda^{m}: \lambda \in \sigma(T)\right\}$. Therefore,

$$
\left\{\lambda^{m}: \lambda \in \sigma(T)\right\}=\{0,1\} \cup\left\{\left(\varphi_{m}^{\prime}\left(z_{0}\right)\right)^{n}: n \in \mathbb{N}\right\}
$$

Corollary 3.4. Suppose that $X$ is a compact plane set with $\operatorname{int}(X) \neq \emptyset, \Omega$ is a connected open set in the complex plane with $\Omega \subseteq \operatorname{int}(X)$, and $K=\bar{\Omega}$. Take $\alpha \in(0,1]$. Let $\varphi$ be a self-map of $X$ with $\varphi(X) \subseteq \Omega$ such that $\varphi \in \operatorname{Lip}_{A}(X, K, \alpha)$, and let $\varphi\left(z_{0}\right)=z_{0}$ for some $z_{0} \in \Omega$. If $T$ is a unital endomorphism of Lip $_{A}(X, K, \alpha)$ induced by $\varphi$, then $T$ is compact and

$$
\sigma(T)=\{0,1\} \cup\left\{\left(\varphi^{\prime}\left(z_{0}\right)\right)^{n}: n \in \mathbb{N}\right\} .
$$

\section{References}

[1] F. Behrouzi and H. Mahyar, "Compact endomorphisms of certain analytic Lipschitz algebras," Bulletin of the Belgian Mathematical Society, vol. 12, no. 2, pp. 301-312, 2005.

[2] T. W. Gamelin, Uniform Algebras, Chelsea Publishing, New York, NY, USA, 1984.

[3] D. R. Sherbert, "Banach algebras of Lipschitz functions," Pacific Journal of Mathematics, vol. 13, pp. 1387-1399, 1963.

[4] D. R. Sherbert, "The structure of ideals and point derivations in Banach algebras of Lipschitz functions," Transactions of the American Mathematical Society, vol. 111, pp. 240-272, 1964.

[5] T. G. Honary and S. Moradi, "On the maximal ideal space of extended analytic Lipschitz algebras," Quaestiones Mathematicae, vol. 30, no. 3, pp. 349-353, 2007.

[6] K. Jarosz, "Lip ${ }_{H o l}(X, \alpha)$," Proceedings of the American Mathematical Society, vol. 125, no. 10, pp. 31293130, 1997.

[7] C. Caratheodory, Theory of Functions of a Complex Variable, vol. 2, Chelsea Publishing, New York, NY, USA, 1960.

[8] J. B. Conway, Functions of One Complex Variable, vol. 11 of Graduate Texts in Mathematics, Springer, New York, NY, USA, 2nd edition, 1978.

[9] H. Kamowitz, "Compact endomorphisms of Banach algebras," Pacific Journal of Mathematics, vol. 89, no. 2, pp. 313-325, 1980. 


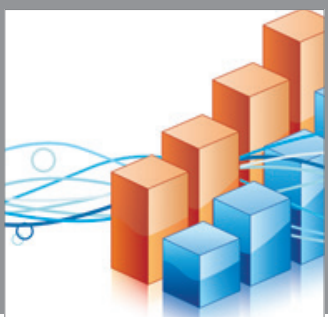

Advances in

Operations Research

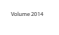

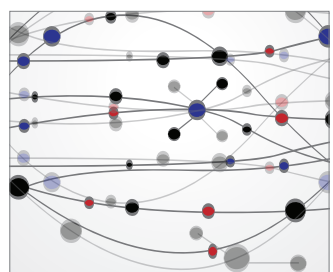

\section{The Scientific} World Journal
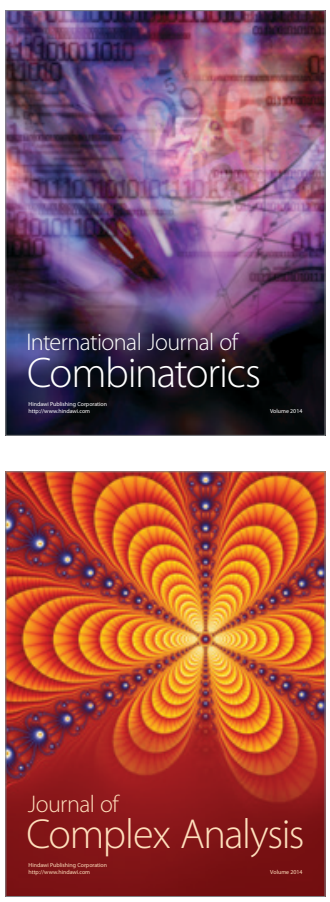

International Journal of

Mathematics and

Mathematical

Sciences
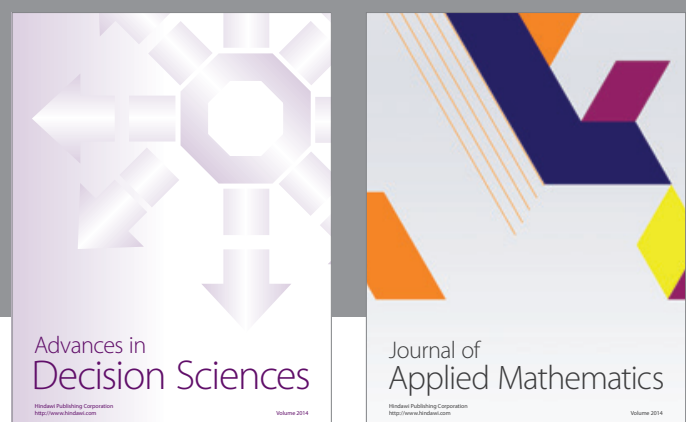

Journal of

Applied Mathematics
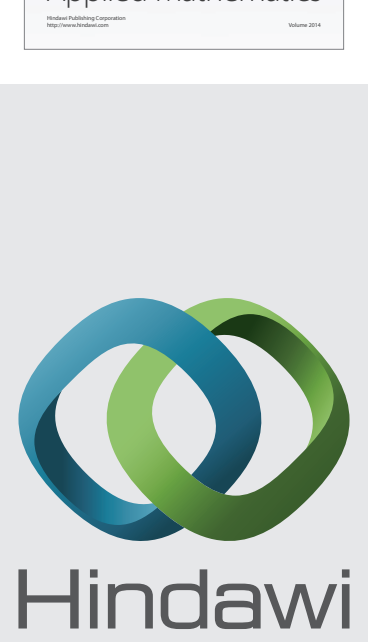

Submit your manuscripts at http://www.hindawi.com
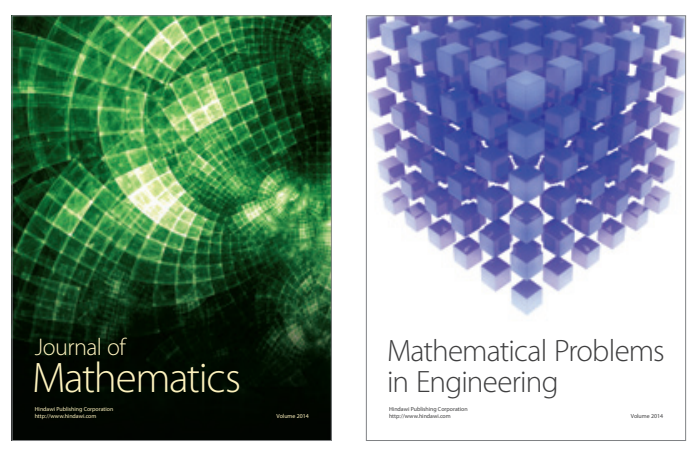

Mathematical Problems in Engineering
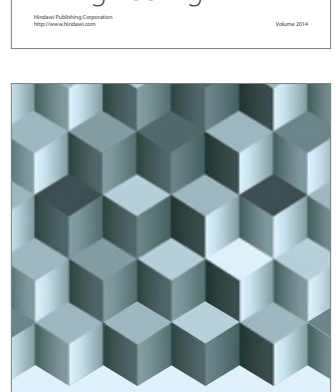

Journal of

Function Spaces
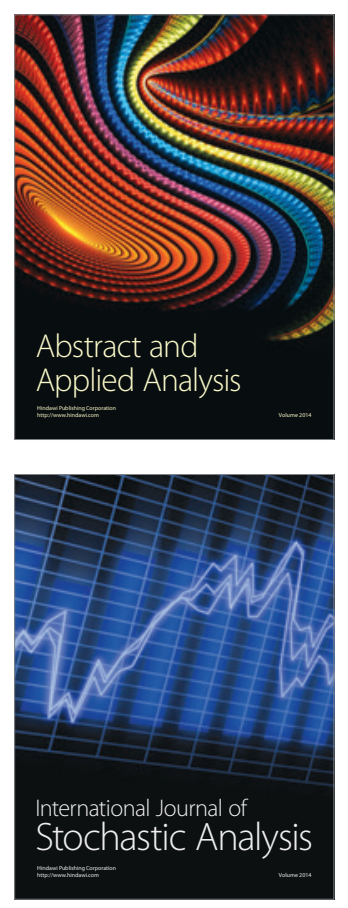

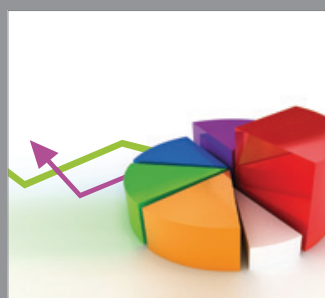

ournal of

Probability and Statistics

Promensencen
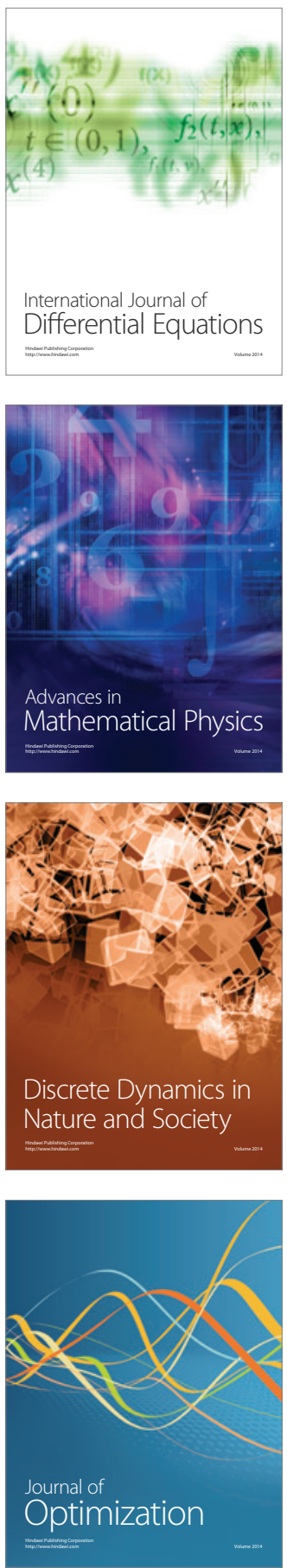